UAHEP 9811

Nov 1998

\title{
Hamiltonian Approach to Poisson Lie T-Duality
}

\author{
A. STERN \\ Dept. of Physics and Astronomy, Univ. of Alabama, Tuscaloosa, Al 35487, U.S.A.
}

\begin{abstract}
The Hamiltonian formalism offers a natural framework for discussing the notion of Poisson Lie T-duality. This is because the duality is inherent in the Poisson structures alone and exists regardless of the choice of Hamiltonian. Thus one can pose alternative dynamical systems possessing nonabelian T-duality. As an example, we find a dual Hamiltonian formulation of the $O(3)$ nonlinear $\sigma$-model. In addition, we easily recover the known dynamical systems having Poisson Lie T-duality starting from a general quadratic Hamiltonian.
\end{abstract}




\section{Introduction}

Poisson Lie T-duality [1], [2], [3], [4] is a nonabelian generalization of Abelian T-duality which shares many structural features with the Abelian system. Unlike in other approaches [5], it does not require the existence of an isometry group. The formulation of Poisson Lie T-duality given so far is tied to an action principle. It therefore implies a particular dynamics, which is namely that of a $\sigma$-model on a group manifold in a background obeying certain geometrical criteria. Thus duality links not only different backgrounds, but different target space manifolds, corresponding to different Lie groups.

The associated Hamiltonian descriptions of the dual systems were derived recently by Sfetsos [6]. There it was shown that the transformations between systems are canonical transformations. Therefore in the Hamiltonian framework, one sees that Poisson Lie T-duality can be introduced independently of the dynamics, as it is a feature of the Poisson algebra alone. One should then be able to pose alternative dual dynamical systems. We wish to explore that possibility here.

For Poisson Lie T-duality one introduces the Drinfeld double group $D$, which by definition has a pair $(G, H)$ of maximally isotropic subgroups[]]. The relevant Poisson algebra for the theory is $\widehat{L D}$, the central extension of the loop group of $D$. [6] This algebra can be realized on either $L T^{*} G$ or $L T^{*} H$, the loop algebras of $T^{*} G$ or $T^{*} H$, respectively. For that reason one can consider $L T^{*} G$ and $L T^{*} H$ as being 'dual'. The duality will persist for any Hamiltonian dynamics we introduce on $\widehat{L D}$. Arbitrary choices for the Hamiltonian may, in general, lead to exotic dynamics, which need not be Lorentz invariant or local. On the other hand, as we shall show, by restricting to quadratic Hamiltonians $\mathrm{H}$ on $\widehat{L D}$ and demanding Lorentz invariant dynamics, more specifically, the dynamics of nonchiral scalar fields, one is able to recover the known systems. The dual Hamiltonians derived in [6] are then recovered by projecting $\mathrm{H}$ onto $L T^{*} G$ and $L T^{*} H$.

Concerning alternative dynamical systems, three approaches can be explored: 1) Instead of utilizing the background fields on $G$ given in [1], one is free to choose the backgrounds arbitrarily. In general, however, it may not be possible to simultaneously have local expressions for the backgrounds on both $G$ and $H$. 2) Instead of restricting to quadratic Hamiltonians, one can examine the possibility of higher order terms. 3) Gauge symmetries can be introduced by imposing first class constraints. The latter must generate a subgroup of $\widehat{L D}$. The target manifolds are now coset spaces, and thus one ends up with dual Hamiltonian descriptions of coset models. We will briefly examine this last approach for the case of the $O(3)$ nonlinear $\sigma$-model.

The outline for this article is as follows: In Sec. 2, we give a simple construction of the duality between Poisson algebras $L T^{*} G$ and $L T^{*} H$. In Sec. 3, we examine a general quadratic Hamiltonian on $\widehat{L D}$, and give its projection onto $L T^{*} G$ and $L T^{*} H$. We then demonstrate how to recover the known dynamics for the Abelian and nonabelian cases in Sec's 4 and 5. We 
examine the case of the $O(3)$ nonlinear $\sigma$-model in Sec. 6 .

\section{Duality of Poisson algebras}

In defining the Drinfeld double group $D$, and its maximally isotropic subgroups $G$ and $H$, say $G$ and $H$ are $n$ dimensional generated by $e_{i}$ and $e^{i}, i=1,2, \ldots n$, respectively, their union generating all of $D$. By definition the commutation relations and invariant scalar product can be written in the form[0]

$$
\begin{aligned}
{\left[e_{i}, e_{j}\right] } & =c_{i j}^{k} e_{k} \\
{\left[e^{i}, e^{j}\right] } & =c_{k}^{i j} e^{k} \\
{\left[e^{i}, e_{j}\right] } & =c_{j k}^{i} e^{k}-c_{j}^{i k} e_{k}, \\
<e^{i} \mid e_{j}>=\delta_{j}^{i}, \quad & <e^{i}\left|e^{j}>=<e_{i}\right| e_{j}>=0 .
\end{aligned}
$$

The phase space of interest is spanned by fields $v(x)=e^{i} v_{i}(x)+e_{i} v^{i}(x)$ taking values in the Lie-algebra associated with $D, x$ being coordinates of a one dimensional spatial domain. As stated in the introduction, the relevant Poisson algebra is the central extension of the loop group of $D[6]$ :

$$
\{\underset{1}{v(x)} \underset{2}{v(y)}\}=[C, v(x)] \delta(x-y)-C \partial_{x} \delta(x-y),
$$

where we use tensor product notation, the 1 and 2 labels referring to two separate vector spaces, with $v(x)=v(x) \otimes 11, v(y)=11 \otimes v(y)$, and 1 being the unit operator acting on the 1

vector spaces. $C$ in (2.2) is a constant adjoint invariant tensor and hence $[C, v(x)+v(x)]=0$. We normalize it according to: $C=e^{i} \otimes e_{i}+e_{i} \otimes e^{i}$.

The algebra (2.2) can be realized on either $L T^{*} G$ or $L T^{*} H$, giving two dual descriptions of the phase space. The former is spanned by the fields $g(x)$ and $J_{i}(x), g(x)$ taking values in $G$, while $J_{i}(x)$ generate left translations on $G$, i.e.

$$
\begin{aligned}
\left\{J_{i}(x), g(y)\right\} & =e_{i} g(x) \delta(x-y) \\
\left\{J_{i}(x), J_{j}(y)\right\} & =-c_{i j}^{k} J_{k}(x) \delta(x-y) .
\end{aligned}
$$

In terms of these variables, $v$ is given by

$$
v=v\left(g, J_{i}\right)=g^{-1} e^{i} g J_{i}+g^{-1} \partial_{x} g .
$$

A straightforward calculation using (2.3) and (2.4) yields

$$
\{v(x), v(y)\}=\underset{1}{g}{ }_{2}(x)^{-1} g(y)^{-1}\left(\left[C, e^{i}\right] J_{i}(x) \delta(x-y)-C \partial_{x} \delta(x-y)\right) g(x) g(y) .
$$


To arrive at (2.2) one can simply apply the identity

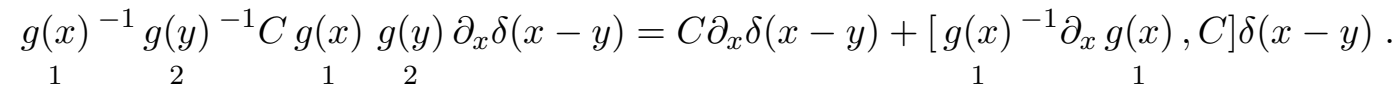

This procedure can be repeated to obtain the dual description associated with $L T^{*} H$. The latter is spanned by the fields $h(x)$ and $\tilde{J}^{i}(x), h(x)$ taking values in $H$, while $\tilde{J}^{i}(x)$ generate left translations on $H$. The analogue of (2.3) is

$$
\begin{aligned}
\left\{\tilde{J}^{i}(x), h(y)\right\} & =e^{i} h(x) \delta(x-y) \\
\left\{\tilde{J}^{i}(x), \tilde{J}^{j}(y)\right\} & =-c_{k}^{i j} \tilde{J}^{k}(x) \delta(x-y) .
\end{aligned}
$$

In terms of these variables, $v$ is given by

$$
v=v\left(h, \tilde{J}^{i}\right)=h^{-1} e_{i} h \tilde{J}^{i}+h^{-1} \partial_{x} h .
$$

From the above it is evident that $L T^{*} G$ and $L T^{*} H$ have a common sector, i.e. $\widehat{L D}$. Topology clearly prevents us, in general, from making a complete identification of the dual descriptions.

For the case where both $G$ and $H$, the duality transformation has a simple form. If we parametrize $g \in G$ and $h \in H$ according to $g=\exp \left(e_{i} \phi^{i}\right)$ and $h=\exp \left(e^{i} \chi_{i}\right)$, we get a nonlocal map between the canonically conjugate field variables $\left(\phi^{i}, J_{i}\right)$ and their duals $\left(\chi_{i}, \tilde{J}^{i}\right)$ :

$$
\begin{aligned}
J_{i}(x) & \rightarrow \partial_{x} \chi_{i}(x) \\
\partial_{x} \phi^{i}(x) & \rightarrow \tilde{J}^{i}(x),
\end{aligned}
$$

which has the effect of interchanging winding modes with momentum modes. [8] For the case of nonabelian $G$ and $H$, if we write

$$
\begin{aligned}
g e_{i} g^{-1} & =a(g)_{i}{ }^{j} e_{j} \\
g e^{i} g^{-1} & =b(g)^{i j} e_{j}+a\left(g^{-1}\right)_{j}{ }^{i} e^{j},
\end{aligned}
$$

and

$$
\begin{aligned}
h e^{i} h^{-1} & =\tilde{a}(h)^{i}{ }_{j} e^{j} \\
h e_{i} h^{-1} & =\tilde{b}(h)_{i j} e^{j}+\tilde{a}\left(h^{-1}\right)^{j}{ }_{i} e_{j},
\end{aligned}
$$

which follow from the Lie algebra (2.1), a duality transformation amounts to making the replacement:

$$
\begin{aligned}
a(g)_{i}{ }^{j} J_{j} & \rightarrow \tilde{b}\left(h^{-1}\right)_{i j} \tilde{J}^{j}+\left(h^{-1} \partial_{x} h\right)_{i} \\
b\left(g^{-1}\right)^{i j} J_{j}+\left(g^{-1} \partial_{x} g\right)^{i} & \rightarrow \tilde{a}(h)^{i}{ }_{j} \tilde{J}^{j} .
\end{aligned}
$$

In general, local descriptions in one theory can become nonlocal in the other. To avoid nonlocal terms in the Hamiltonian $\mathrm{H}$ after projecting onto $L T^{*} G$ or $L T^{*} H$, below we will 
restrict to the case where $\mathrm{H}$ is a (local) function on $\widehat{L D}$. Starting from general quadratic systems, we shall show how the dynamics for free massless scalar fields can be recovered for the case of Abelian T-duality, and also how the dynamical system of Klimcik and Severa[1] can be recovered in the nonabelian case.

\section{Dual Dynamics}

To recover known systems, we examine Hamiltonians which are quadratic in $v$. The most general such Hamiltonian is

$$
\mathrm{H}=\frac{1}{2} \int d x<v \mid R v>,
$$

where $R$, for the moment, is an arbitrary linear operator. It is easy to compute the resulting Hamilton equations. Using (2.2), we get

$$
\begin{aligned}
& \{v(x), \mathrm{H}\}=\int d y<\left.\begin{array}{ccc}
v(x), v(y) \\
1
\end{array} \underset{2}{2}\right|_{2} v(y)>_{2} \\
& =-<[C, v(x)] \mid R \quad v(x)>_{2}-<\underset{2}{R} \quad \begin{array}{c}
R \partial_{x} v(x) \\
2
\end{array}>_{2}
\end{aligned}
$$

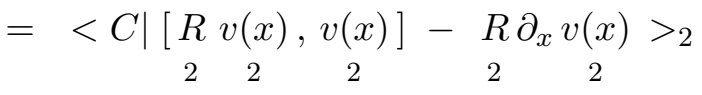

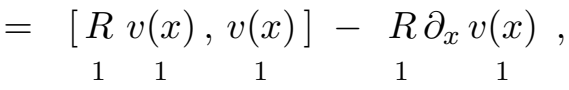

where $<,>_{2}$ denotes a scalar product with respect to the second vector space in the tensor product. Thus

$$
\partial_{t} v=-R \partial_{x} v+[R v, v]
$$

which is the Muarer-Cartan equation on $D$ once we identify $v(x)$ and $-R v(x)$ with the space and time components, respectively, of a one form $V$ on a two dimensional space-time domain. Then, at least on an open coordinate patch of the domain, we can write $V=\ell^{-1} d \ell, \ell \in D$. This yields

$$
\ell^{-1} \partial_{t} \ell+R\left(\ell^{-1} \partial_{x} \ell\right)=0
$$

These equations of motion have the same form as the defining equations of Klimcik and Severa [1]. The only difference is that we have not yet specified $R$. Moreover, it was found [2], [3], [4] that the dynamical system of [1] could be obtained starting from an action principle on $D$. The action corresponds to the Wess-Zumino-Witten model (written on the light cone), minus the term (3.1), with a particular definition of the linear operator $R$. The Wess-ZuminoWitten model (written on the light cone) defines the symplectic two-form for the theory. 
It corresponds precisely to the Poisson algebra (2.2). The remaining term then defines the Hamiltonian and thus also agrees with what we wrote (except for us, $R$ is arbitrary).

Without any loss of generality, we can write $R$ such that

$$
\begin{aligned}
R e_{i} & =-\mathcal{F}_{i j}^{0} e^{j}-\mathcal{H}^{0}{ }_{i}{ }^{j} e_{j} \\
R e^{i} & =-\mathcal{H}^{0}{ }_{j}{ }_{j} e^{j}-\mathcal{G}^{0 i j} e_{j},
\end{aligned}
$$

where $\mathcal{F}^{0}, \mathcal{G}^{0}$ and $\mathcal{H}^{0}$ are constant matrices, the first two being symmetric. Then

$$
\mathrm{H}=-\frac{1}{2} \int d x\left(\mathcal{F}_{i j}^{0} v^{i} v^{j}+\mathcal{G}^{0 i j} v_{i} v_{j}+2 \mathcal{H}^{0}{ }_{i}{ }^{j} v^{i} v_{j}\right)
$$

and using (2.4) or (2.8), we can express the Hamiltonian on either $L T^{*} G$ or $L T^{*} H$ as follows:

$$
\begin{aligned}
& \mathrm{H}\left(g, J^{i}\right)=-\frac{1}{2} \int d x\left(\mathcal{F}(g)_{i j}\left(\partial_{x} g g^{-1}\right)^{i}\left(\partial_{x} g g^{-1}\right)^{j}+\mathcal{G}(g)^{i j} J_{i} J_{j}+2 \mathcal{H}(g)_{i}{ }^{j}\left(\partial_{x} g g^{-1}\right)^{i} J_{j}\right), \\
& \mathrm{H}\left(h, \tilde{J}_{i}\right)=-\frac{1}{2} \int d x\left(\tilde{\mathcal{F}}(h)_{i j} \tilde{J}^{i} \tilde{J}^{j}+\tilde{\mathcal{G}}(h)^{i j}\left(\partial_{x} h h^{-1}\right)_{i}\left(\partial_{x} h h^{-1}\right)_{j}+2 \tilde{\mathcal{H}}(h)_{i}{ }^{j} \tilde{J}^{i}\left(\partial_{x} h h^{-1}\right)_{j}\right),
\end{aligned}
$$

respectively. To evaluate the functions $\mathcal{F}(g), \mathcal{G}(g)$ and $\mathcal{H}(g)$ we can first use (2.10) to write

$$
\begin{aligned}
v^{i} & =a\left(g^{-1}\right)_{j}{ }^{i}\left(\partial_{x} g g^{-1}\right)^{j}+b(g)^{i j} J_{j}, \\
v_{i} & =a(g)_{i}{ }^{j} J_{j} .
\end{aligned}
$$

Then we can substitute into (3.6) to find

$$
\begin{aligned}
\mathcal{F}(g) & =a(g)^{-1} \mathcal{F}^{0} a(g)^{-1} \\
\mathcal{H}(g) & =a(g)^{-1} \mathcal{F}^{0} b(g)+a(g)^{-1} \mathcal{H}^{0} a(g) \\
\mathcal{G}(g) & =b(g)^{T} \mathcal{F}^{0} b(g)+a(g)^{T} \mathcal{G}^{0} a(g)+b(g)^{T} \mathcal{H}^{0} a(g)+a(g)^{T} \mathcal{H}^{0^{T}} b(g) .
\end{aligned}
$$

Similarly, by writing

$$
\begin{aligned}
v^{i} & =\tilde{a}(h)^{i}{ }_{j} \tilde{J}^{j} \\
v_{i} & =\tilde{a}\left(h^{-1}\right)^{j}{ }_{i}\left(\partial_{x} h h^{-1}\right)_{j}+\tilde{b}(h)_{i j} \tilde{J}^{j},
\end{aligned}
$$

which follows from (2.11), $\tilde{\mathcal{F}}(h), \tilde{\mathcal{G}}(h)$ and $\tilde{\mathcal{H}}(h)$ are given by:

$$
\begin{aligned}
\tilde{\mathcal{F}}(h) & =\tilde{b}(h)^{T} \mathcal{G}^{0} \tilde{b}(h)+\tilde{a}(h)^{T} \mathcal{F}^{0} \tilde{a}(h)+\tilde{b}(h)^{T} \mathcal{H}^{0^{T}} \tilde{a}(h)+\tilde{a}(h)^{T} \mathcal{H}^{0} \tilde{b}(h) \\
\tilde{\mathcal{H}}(h) & =\tilde{b}(h)^{T} \mathcal{G}^{0} \tilde{a}(h)^{-1^{T}}+\tilde{a}(h)^{T} \mathcal{H}^{0} \tilde{a}(h)^{-1^{T}} \\
\tilde{\mathcal{G}}(h) & =\tilde{a}(h)^{-1} \mathcal{G}^{0} \tilde{a}(h)^{-1^{T}} .
\end{aligned}
$$

In general, for an arbitrary choice of $R$, and hence the constant matrices $\mathcal{F}^{0}, \mathcal{G}^{0}$ and $\mathcal{H}^{0}$, the equations of motion (3.4) may not be Lorentz invariant. On the other hand, we can require that the system leads to familiar dynamics, thereby specifying $R$. Below we shall restrict to the system of nonchiral massless scalar fields, and thereby recover known dynamical systems possessing T-duality. We begin with the Abelian case. 


\section{Abelian case}

If both $G$ and $H$ are Abelian, the backgrounds $[\mathcal{F}(g), \mathcal{G}(g), \mathcal{H}(g)]$ and $[\tilde{\mathcal{F}}(h), \tilde{\mathcal{G}}(h), \tilde{\mathcal{H}}(h)]$ reduce to the constant matrices $\left[\mathcal{F}^{0}, \mathcal{G}^{0}, \mathcal{H}^{0}\right]$. Furthermore, the equations of motion (3.4) reduce to

$$
\partial_{t} \psi+R \partial_{x} \psi=0
$$

where $\psi=\phi^{i} e_{i}+\chi_{i} e^{i}$ is an element of the Lie algebra of $D$. Then

$$
\begin{aligned}
\partial_{t} \chi_{i} & =\mathcal{H}^{0}{ }_{i}{ }^{j} \partial_{x} \chi_{j}+\mathcal{F}_{i j}^{0} \partial_{x} \phi^{j} \\
\partial_{t} \phi^{i} & =\mathcal{G}^{0 i j} \partial_{x} \chi_{j}+\mathcal{H}^{0}{ }_{j}{ }_{j}{ } \partial_{x} \phi^{j}
\end{aligned}
$$

For the simplest case of $n=1$, these equations imply the existence of a pair of plane wave solutions with velocities determined by the three constants $\mathcal{H}^{0}, \mathcal{G}^{0}$ and $\mathcal{F}^{0}$

$$
\left[\partial_{t}-\left(\mathcal{H}^{0} \pm \sqrt{\mathcal{G}^{0} \mathcal{F}^{0}}\right) \partial_{x}\right] \psi^{ \pm}=0, \quad \psi^{ \pm}=\chi \pm \sqrt{\mathcal{F}^{0} / \mathcal{G}^{0}} \phi
$$

provided $\mathcal{G}^{0} \neq 0$. For arbitrary values of the constants we will not get Lorentz invariant dynamics. To recover left and right moving light-like waves we need $\mathcal{H}^{0}=0$ and $\mathcal{G}^{0}=1 / \mathcal{F}^{0}$. Eqs. (3.7) and (3.8) then reduce to the standard dual Hamiltonians for a massless scalar field, with the duality transformation corresponding to the familiar $\mathcal{G}^{0} \rightarrow 1 / \mathcal{G}^{0}$. [8] [Alternatively, a chiral system with only right moving light-like waves results from the choice $\mathcal{H}^{0}=1$ and $\mathcal{G}^{0}=\mathcal{F}^{0}=0$. In this case, however, the system is self-dual, as then the expressions for the Hamiltonians (3.7) and (3.8) are identical.]

For the case where $n$ is arbitrary, let us demand that (4.2) describes $n$ nonchiral massless fields. Then $R$ should have eigenvalues \pm 1 with eigenvectors of the form $\psi=\phi^{i} e_{i}+\chi_{i}[\phi] e^{i}$, where $\chi_{i}$ are determined from $\phi^{i}$, and $\phi^{i}$ are arbitrary (or vice versa). This is possible provided that $\mathcal{G}^{0}$ is nonsingular and

$$
\mathcal{F}^{0}=\left(\mathcal{H}^{0}+11\right) \mathcal{G}^{0-1}\left(\mathcal{H}^{0}+11\right)^{T}=\left(\mathcal{H}^{0}-11\right) \mathcal{G}^{0-1}\left(\mathcal{H}^{0}-11\right)^{T}
$$

This then implies that the matrix $\mathcal{B}^{0}=\mathcal{H}^{0} \mathcal{G}^{0-1}$ is antisymmetric. We denote by $\left\{T_{\alpha}^{ \pm}, i=\right.$ $1,2, \ldots n, \alpha= \pm\}$ the eigenvectors of $R$

$$
R T_{i}^{\alpha}=\alpha T_{i}^{\alpha}
$$

They are given by

$$
T_{i}^{ \pm}=e_{i}-\mathcal{E}_{i j}^{ \pm} e^{j}, \quad \mathcal{E}_{i j}^{ \pm}= \pm \mathcal{G}_{i j}^{0}+\mathcal{B}_{i j}^{0},
$$

where $\mathcal{G}_{i j}^{0}$ are the matrix elements of $\mathcal{G}^{0-1}$, and have inner product $<T_{i}^{\alpha} \mid T_{j}^{\beta}>=-2 \alpha \delta_{\alpha, \beta} \mathcal{G}_{i j}^{0}$. By substituting the above result for the constant matrices, i.e.

$$
\begin{aligned}
\mathcal{F}_{i j}^{0} & =\mathcal{G}_{i j}^{0}-\mathcal{B}_{i k}^{0} \mathcal{G}^{0 k \ell} \mathcal{B}_{\ell j}^{0} \\
\mathcal{H}^{0}{ }_{i}{ }^{j} & =\mathcal{B}_{i k}^{0} \mathcal{G}^{0 k j}
\end{aligned}
$$


into (4.2), we get the standard equations of motion for massless modes familiar in string theory [9] and also in the quantum Hall effect [1].

In addition to the two dual descriptions, described in the previous section, it is well known (in the Abelian case) that there is an entire family of dual descriptions related by $O(n, n)$ canonical transformations [12] [11]:

$$
\left(\begin{array}{l}
v^{i} \\
v_{i}
\end{array}\right) \rightarrow \mathcal{O}\left(\begin{array}{l}
v^{i} \\
v_{i}
\end{array}\right), \quad \mathcal{O}\left(\begin{array}{ll}
11 \\
11
\end{array}\right) \mathcal{O}^{T}=\left(\begin{array}{ll} 
& 11 \\
11 &
\end{array}\right) .
$$

Topological and quantum considerations further restrict the transformations to a discrete subgroup corresponding to $O(n, n, Z)$. Under such transformations, the constant matrices $\mathcal{H}^{0}, \mathcal{G}^{0}$ and $\mathcal{F}^{0}$ in the Hamiltonian are transformed by

$$
\left(\begin{array}{cc}
\mathcal{F}^{0} & \mathcal{H}^{0} \\
\mathcal{H}^{0} & \mathcal{G}^{0}
\end{array}\right) \rightarrow \mathcal{O}^{T}\left(\begin{array}{cc}
\mathcal{F}^{0} & \mathcal{H}^{0} \\
\mathcal{H}^{0} & \mathcal{G}^{0}
\end{array}\right) \mathcal{O}
$$

\section{Nonabelian case}

The nonabelian generalization of Abelian T-duality considered by Klimcik and Severa[1] utilizes the same definition of $R$ as in the Abelian case, i.e. (4.5) and (4.6). At the level of the equations of motion, duality was achieved after projecting (3.4) onto $G$ and $H$. We now review the procedure.

By parametrizing the Drinfeld double variables according to $\ell=\tilde{h} g, \tilde{h} \in H$ and $g \in G$, one gets

$$
\ell^{-1} d \ell=g^{-1} \tilde{h}^{-1} d \tilde{h} g+g^{-1} d g .
$$

Comparing with (2.4) we see that $J_{i} e^{i}$ is identified with $\tilde{h}^{-1} \partial_{x} \tilde{h}$. Eq. (3.4) can be expressed as

$$
<T_{i}^{ \pm} \mid \ell^{-1} \partial_{ \pm} \ell>=0, \quad \partial_{ \pm}=\partial_{t} \pm \partial_{x}
$$

and hence

$$
<g T_{i}^{ \pm} g^{-1} \mid A_{ \pm i} e^{i}+A_{ \pm}^{i} e_{i}>=0
$$

where $A_{ \pm}^{i} e_{i}=\partial_{ \pm} g g^{-1}, A_{ \pm i} e^{i}=\tilde{h}^{-1} \partial_{ \pm} \tilde{h}$, and we used the invariance property of the inner product. To evaluate (5.3), we can use (2.10) and express $g T_{i}^{ \pm} g^{-1}$ according to

$$
\begin{aligned}
g T_{i}^{+} g^{-1} & =M^{+}(g)_{i}{ }^{j}\left[e_{j}-E(g)_{j k} e^{k}\right] \\
g T_{i}^{-} g^{-1} & =M^{-}(g)_{i}{ }^{j}\left[e_{j}+E(g)_{k j} e^{k}\right]
\end{aligned}
$$

where

$$
M^{ \pm}(g)=a(g)-\mathcal{E}^{ \pm} b(g)
$$

and

$$
E(g)=M^{+}(g)^{-1} \mathcal{E}^{+} a(g)^{T^{-1}} .
$$


Equations (5.3) become

$$
\begin{aligned}
& A_{+i}-E(g)_{i j} A_{+}^{j}=0 \\
& A_{-i}+E(g)_{j i} A_{-}^{j}=0 .
\end{aligned}
$$

These equations define the connection components $A_{ \pm i}$ as functions on $T G$. They satisfy the Maurer-Cartan equations associated with $H$,

$$
\partial_{-} A_{+i}-\partial_{+} A_{-i}+c_{i}^{j k} A_{j-} A_{k+}=0 .
$$

These equations were obtained from an action principle written on $T G$, the Lagrangian density being

$$
L=E(g)_{i j} A_{-}^{i} A_{+}^{j} .
$$

Eq. (5.8) results from extremizing $\int d^{2} x L$ in $g$, using the definition (5.6) of the background $E(g)$. In addition, we have the Maurer-Cartan equations associated with $G$,

$$
\partial_{-} A_{+}^{i}-\partial_{+} A_{-}^{i}-c_{j k}^{i} A_{-}^{j} A_{+}^{k}=0
$$

which here play the role of identities. The Hamiltonian associated with the Lagrangian (5.9) is written on $L T^{*} G$ and was derived in [6]. It is just (3.7) with constant matrices given by (4.7).

The dual description is obtained by instead factorizing the Drinfeld double variables according to $\ell=\tilde{g} h, h \in H$ and $\tilde{g} \in G$, and repeating the above procedure. Now from (2.8), $\tilde{J}^{i} e_{i}$ is identified with $\tilde{g}^{-1} \partial_{x} \tilde{g}$. Using (2.11), the analogue of the matrix $E(g)$ is

$$
\tilde{E}(h)=\left[\tilde{a}(h)-\mathcal{E}^{+-1} \tilde{b}(h)\right]^{-1} \mathcal{E}^{+-1} \tilde{a}(h)^{T^{-1}} .
$$

Defining $\tilde{A}_{ \pm i} e^{i}=-\partial_{ \pm} h h^{-1}, \tilde{A}_{ \pm}^{i} e_{i}=-\tilde{g}^{-1} \partial_{ \pm} \tilde{g}$, the equations (5.3) can now be written as

$$
\begin{aligned}
& \tilde{A}_{+}^{i}-\tilde{E}(h)^{i j} \tilde{A}_{+j}=0 \\
& \tilde{A}_{-}^{i}+\tilde{E}(h)^{j i} \tilde{A}_{-j}=0
\end{aligned}
$$

stating that the connection components $\tilde{A}_{ \pm}^{i}$ are functions on $T H$. They satisfy the MaurerCartan equations associated with $G$,

$$
\partial_{-} \tilde{A}_{+}^{i}-\partial_{+} \tilde{A}_{-}^{i}-c_{j k}^{i} \tilde{A}_{-}^{j} \tilde{A}_{+}^{k}=0 .
$$

This is obtainable from an action principle written on $T H$, the Lagrangian density being

$$
\tilde{L}=\tilde{E}(h)^{i j} \tilde{A}_{i-} \tilde{A}_{j+} .
$$

Eq. (5.13) results from extremizing $\int d^{2} x \tilde{L}$ in $h$, using the definition (5.11) of the background $\tilde{E}(h)$. In addition, we have the Maurer-Cartan equations associated with $H$

$$
\partial_{-} \tilde{A}_{+i}-\partial_{+} \tilde{A}_{-i}+c_{i}^{j k} \tilde{A}_{j-} \tilde{A}_{k+}=0
$$

which now are identities. Thus the roles of identities and equations of motion are reversed in the two descriptions. The Hamiltonian associated with the Lagrangian (5.14) is written on $L T^{*} H$ and is just (3.7) with constant matrices again given by (4.7). 


\section{$6 \quad O(3)$ nonlinear $\sigma$-model}

We have argued that Poisson Lie T-duality need not be tied to any particular dynamics on $G$ or $H$. By making relatively simple assumptions on the Hamiltonian, we have shown how to recover the known dynamical systems having Poisson Lie T-duality. But alternative dynamics should also be of interest. As an example, here we consider modifying the dynamics by introducing gauge symmetries. This will allow us to describe coset models. Here we will just examine the simple case of the $O(3)$ nonlinear $\sigma$-model.

In the Hamiltonian formulation we can introduce gauge symmetries by imposing first class constraints. The latter should generate a subgroup of $\widehat{L D}$. For example, one can set one component of $v$ (weakly) equal to zero, say $v_{1}$. The resulting gauge invariant physical subspace will be $2 n-2$ dimensional. Upon projecting the constraint onto $L T^{*} G$, we get

$$
\Phi\left(g, J_{i}\right)=a(g)_{1}^{i} J_{i} \approx 0
$$

generating right transformations on $G: \delta g=\epsilon g e_{1}, \epsilon$ being infinitesimal. On $L T^{*} H$ the constraint will look like

$$
\tilde{\Phi}\left(h, \tilde{J}^{i}\right)=\tilde{b}(h)_{1 i} \tilde{J}^{i}+\left[\tilde{a}\left(h^{-1}\right)\right]_{1}^{i}\left(\partial_{x} h h^{-1}\right)_{i} \approx 0,
$$

generating a more complicated set of orbits on $H: \delta h=\epsilon \tilde{b}(h)_{1 i} e^{i} h$.

Let us now specialize to the case where $D=T^{*} S U(2)$, with $G=S U(2)$ and $H$ is the three dimensional Abelian group. This has been referred to as the semiabelian case. Then $c_{i j}^{k}=\epsilon_{i j k}$ and $c_{k}^{i j}=0, i, j, k, . .=1,2,3$. From (2.10) and (2.11) it follows that

$$
b(g)^{i j}=0, \quad \tilde{a}(h)^{i}{ }_{j}=\delta_{j}^{i}, \quad \tilde{b}(h)_{i j}=\epsilon_{i j k} \chi_{k},
$$

where we write $h=\exp \left(\chi_{i} e^{i}\right)$. Once we impose the constraint (6.1) on $L T^{*} G$ we are left with four gauge invariant field degrees of freedom. The corresponding configuration space is spanned by fields $\psi^{i}(x)$ having values in $S U(2) / U(1)$, the target space for the $O(3)$ nonlinear $\sigma$-model. $\psi^{i}(x)$ satisfy the constraint $\psi^{i}(x) \psi^{i}(x)=1$, and can be defined by

$$
\psi^{i}(x) e_{i}=g(x) e_{1} g(x)^{-1}
$$

$g(x)$ having values in $S U(2)$. The standard Lagrangian density is 13.

$$
L=\frac{1}{2} \partial_{\mu} \psi^{i} \partial^{\mu} \psi^{i}=\frac{1}{2}\left(g^{-1} \partial_{\mu} g\right)_{a}\left(g^{-1} \partial^{\mu} g\right)_{a}, \quad a=2,3
$$

where $\mu$ is the space time index. It is straightforward to derive the corresponding canonical Hamiltonian. Up to a Lagrange multiplier term involving the constraint (6.1), it can be written

$$
\mathrm{H}\left(g, J^{i}\right)=-\frac{1}{2} \int d x\left(\left[a(g)^{-1}\right]_{i}{ }^{a}\left[a(g)^{-1}\right]_{j}{ }^{a}\left(\partial_{x} g g^{-1}\right)^{i}\left(\partial_{x} g g^{-1}\right)^{j}+[a(g)]_{a}{ }^{i}[a(g)]_{a}^{j} J_{i} J_{j}\right) .
$$

$J_{i}$ generate left transformations on $G$ as in (2.3), and there are no secondary constraints. Eq. (6.6) is consistent with having a quadratic Hamiltonian on $\widehat{L D}$ and projecting onto $L T^{*} G$. 
Comparing with $(3.7)$ and $(3.10)$, we can identify the constant matrices $\left[\mathcal{F}^{0}, \mathcal{G}^{0}, \mathcal{H}^{0}\right]$ for this case. The only nonzero components are

$$
\mathcal{F}_{22}^{0}=\mathcal{F}_{33}^{0}=1, \quad \mathcal{G}^{022}=\mathcal{G}^{033}=1
$$

From (2.11) and (3.12) we can then read off the dual Hamiltonian. In terms of the canonically conjugate variables $\chi_{i}$ and $\tilde{J}^{i}$ it is given by:

$$
\mathrm{H}\left(h, \tilde{J}^{i}\right)=-\frac{1}{2} \int d x\left(\tilde{J}^{a} \tilde{J}^{a}+\left(\epsilon_{a i j} \tilde{J}^{i} \chi_{j}+\partial_{x} \chi_{a}\right)^{2}\right),
$$

up to the constraint (6.2), which here reduces to

$$
\tilde{\Phi}\left(h, \tilde{J}^{i}\right)=\epsilon_{1 a b} \tilde{J}^{a} \chi_{b}+\partial_{x} \chi_{1} \approx 0
$$

Eq. (6.8) differs from the dual Hamiltonian of the principal chiral model 14 since the index $a$ is restricted to values 2,3. From (6.9) gauge transformations are associated with rotations about the 1 -axis in the target space spanned by $\chi_{i}$.

Lastly, we note that the above dual formulation of the $O(3)$ nonlinear $\sigma$-model is not unique. This is because there exists at least one other Drinfeld double group $D$ containing containing $S U(2)$ as a maximally isotropic subgroup. It is $D=S L(2, C)$. Here one can take $H=S B(2, C)$. [15] The above procedure can be repeated in this case to find a dual Hamiltonian formulation of the $O(3)$ nonlinear $\sigma$-model written on $L T^{*} S B(2, C)$.

\section{Acknowledgments}

I am grateful to S. Frolov, G. Marmo, F. Lizzi, A. Simoni and P. Vitale for the stimulating discussions. I thank G. Marmo and members of Dipartimento di Scienze Fisiche, Università di Napoli, for their hospitality and support where this work was initiated. A.S. was supported in part by the U.S. Department of Energy under contract number DE-FG05-84ER40141.

\section{REFERENCES}

[1] C. Klimcik and P. Severa, Phys. Lett. B351 (1995) 455, C. Klimcik, Nucl. Phys.(Proc. Suppl.) B46 (1996) 116.

[2] C. Klimcik and P. Severa,Phys. Lett. B372 (1996) 65; Phys. Lett. B376 (1996) 82; Phys. Lett. B383 (1996) 281.

[3] E. Tyurin, R. von Unge, Phys. Lett. B382 (1996) 233.

[4] A. Yu. Alekseev, C. Klimcik and A.A. Tseytlin, Nucl. Phys. B458 (1996) 430.

[5] X. de la Ossa and F. Quevedo, Nucl. Phys. B403 (1993) 377; E. Álvarez, L. ÁlvarezGaumé, J.L.F. Barbón and Y. Lozano, Nucl. Phys. B415 (1994) 71; E. Álvarez, L. 
Álvarez-Gaumé, and Y. Lozano, Nucl. Phys. B424 (1994) 155; A. Giveon and M. Rocek, Nucl. Phys. B421 (1994) 173;S. Elitzur, A. Giveon, E. Rabinovici, A. Schwimmer and G. Veneziano, Nucl. Phys. B435 (1995) 147.

[6] K. Sfetsos, Nucl. Phys. B517 (1998) 549; Nucl. Phys.(Proc. Suppl.) B56 (1997) 302.

[7] V.G. Drinfeld, Quantum Groups, in Proc. ICM, MSRI, Berkeley, 1986, p. 708.

[8] K. Kikkawa and M. Yamasaki, Phys. Lett. B149 (1984) 357; N. Sakai and I. Senda, Prog. Theor. Phys. 75 (1986) 692; V.P. Nair, A. Shapere, A. Strominger and F. Wilczek, Nucl. Phys. B287 (1987) 402; B. Sathiapalan, Phys. Rev. Lett. 58 (1987) 1597; T.H. Buscher, Phys. Lett. B194 (1987) 51; Phys. Lett. B201 (1988) 466.

[9] K.S. Narain, Phys. Lett. B169 (1986) 61; K.S. Narain, H. Sarmadi, E. Witten, Nucl. Phys. B279 (1987) 309.

[10] M.B. Green, J.H. Schwarz and E. Witten, Superstring Theory, (Cambridge University Press, Cambridge, 1989).

[11] A. P. Balachandran, L. Chandar and B. Sathiapalan, Nucl. Phys. B443[FS] (1995) 465; Int. J. of Mod. Phys. A11 (1996) 3587.

[12] A. Shapere and F. Wilczek, Nucl. Phys. B320 (1989) 669; A. Giveon, E. Rabinovici and G. Veneziano, Nucl. Phys. B322 (1989) 167; A. Giveon and M. Rocek, Nucl. Phys. B380 (1992) 128; J. Maharana and J.H. Schwarz, Nucl. Phys. B390 (1993) 3; E. Álvarez, L. Álvarez-Gaumé, and Y. Lozano, Phys. Lett. B336 (1994) 183.

[13] A. P. Balachandran, A. Stern and G. Trahern, Phys. Rev. D19 (1979) 2416; A. P. Balachandran, G. Marmo, B.S. Skagerstam and A. Stern, Classical Topology and Quantum States (World Scientific, Singapore, 1991).

[14] Y. Lozano, Phys. Lett. B355 (1995) 165.

[15] See for example, G. Marmo, S. Simoni and A. Stern, Int. J. of Mod. Phys. A10 (1995) 99. 